\title{
Acta
Biochimica
Polonica
}

Vol. 48 No. 3/2001

$611-614$

QUARTERLY

\section{Detection of alkylation damage in human lymphocyte DNA with the comet assay ${ }^{\oplus}$}

\author{
Andrew R. Collins ${ }^{凶}$, Mária Dušinská ${ }^{\text {and Alexandra Horská }}$ \\ Rowett Research Institute, Aberdeen, U.K.
}

Received: 22 January, 2001; revised: 2 April, 2001; accepted: 7 May, 2001

Key words: comet assay, DNA damage, AlkA

\begin{abstract}
The enzyme 3-methyladenine DNA glycosylase II (AlkA) is a bacterial repair enzyme that acts preferentially at 3-methyladenine residues in DNA, releasing the damaged base. The resulting baseless sugars are alkali-labile, and under the conditions of the alkaline comet assay (single cell gel electrophoresis) they appear as DNA strand breaks. AlkA is not lesion-specific, but has a low activity even with undamaged bases. We have tested the enzyme at different concentrations to find conditions that maximise detection of alkylated bases with minimal attack on normal, undamaged DNA. AlkA detects damage in the DNA of cells treated with low concentrations of methyl methanesulphonate. We also find low background levels of alkylated bases in normal human lymphocytes.
\end{abstract}

Single cell gel electrophoresis (the comet assay) is widely used for the detection of strand breaks in nuclear DNA. It is particularly appropriate for studying the low background levels of damage present in normal human cells, such as peripheral lymphocytes. The cells are embedded in agarose on a microscope slide and lysed with Triton X-100 and $2.5 \mathrm{M} \mathrm{NaCl}$, which remove cytoplasm and most nuclear proteins, but leave the DNA, in supercoiled form, as nucleoids. After incubation in alkali, the DNA is electrophoresed at high $\mathrm{pH}$; DNA is drawn out to form a 'tail' (hence the name 'comet assay') - but only if breaks are present to relax the supercoiling of the nucleoid DNA. In order to increase its sensitivity and selec-

This work was financially supported by the Scottish Executive Rural Affairs Department, and the EC (Inco-Copernicus contract IC15-CT96-1012).

Present address: Institute of Preventive and Clinical Medicine, Limbová 14, 83301 Bratislava, Slovak Republic.

${ }^{\varpi}$ Professor Andrew R. Collins, Rowett Research Institute, Greenburn Road, Bucksburn, Aberdeen AB21 9SB, Scotland, U.K.; tel. (44 1224) 716 634; fax (44 1224) 716 629; e-mail: a.collins@rri.sari.ac.uk

Abbreviations: AlkA, 3-methyladenine DNA glycosylase II; DAPI, 4,6-diamidino-2-phenylindole; FPG, formamidopyrimidine DNA glycosylase; MMS, methyl methanesulphonate; $\mathrm{NaCl} / \mathrm{P}_{\mathrm{i}}$ buffer, phosphate-buffered saline, pH 7.3; NTH, endonuclease III. 
tivity, we have incorporated into the assay an extra step in which the nucleoid DNA is digested with a lesion-specific endonuclease; the additional breaks revealed with this procedure indicate the presence of the particular lesion. So far, endonuclease III (NTH, specific for oxidised pyrimidines) (Collins et al., 1993), formamidopyrimidine DNA glycosylase (FPG, acting on ring-opened purines and the major purine oxidation produce, 8-oxoguanine) (Dušinská \& Collins, 1996) and T4 endonuclease V (recognising UV-induced cyclobutane pyrimidine dimers) (Collins et al., 1997b) have been successfully employed. Amongst other things, we have estimated background levels of DNA oxidation (Collins et al., 1997a), and have found this damage to be elevated in human diseases such as diabetes and ankylosing spondylitis (Dušinská et al., 1999).

We now report the use of AlkA, a bacterial repair enzyme whose main substrate is 3-methyladenine in DNA, though it also recognises - with lower efficiency - other modified bases (Lindahl, 1993). A recent report (Berdal et al., 1998) suggests that repair enzymes supposedly specific for alkylated bases may in fact create breaks non-selectively (though much less efficiently) at normal bases. Given the size of the genome, even a low efficiency of non-specific breakage could significantly interfere in estimations of background levels of alkylation damage. We reasoned that, by employing a range of concentrations of the enzyme, and carrying out incubations for different lengths of time, we might find a concentration at which only the alkylated bases would be detected, so that the number of breaks would increase to a certain level and then plateau. After optimising reaction conditions, we tested the assay on lymphocytes from different individuals, and also, as a positive control, examined alkylation damage induced by methyl methanesulphonate.

\section{MATERIALS AND METHODS}

AlkA enzyme. AlkA was kindly provided by Dr. T. O’Connor (Beckman Research Institute of the City of Hope National Medical Center, Duarte, U.S.A.) in a highly purified state, at a concentration of $0.4 \mathrm{mg} / \mathrm{ml}$.

Single cell gel electrophoresis. Lymphocytes were isolated from $30 \mu \mathrm{l}$ finger-prick samples of blood by standard centrifugation over a cushion of Histopaque, washed with $\mathrm{NaCl} / \mathrm{P}_{\mathrm{i}}$ buffer, and centrifuged. The pellet of cells was suspended in $170 \mu \mathrm{l}$ of low melting point $1 \%$ agarose in $\mathrm{NaCl} / \mathrm{P}_{\mathrm{i}}$ buffer and quickly placed as two $85 \mu \mathrm{l}$ aliquots on a glass microscope slide. Glass cover slips were placed on the gels, which were allowed to set at $4^{\circ} \mathrm{C}$. Cells were lysed by immersing slides in $2.5 \mathrm{M} \mathrm{NaCl}, 0.1 \mathrm{M}$ EDTA, $10 \mathrm{mM}$ Tris/HCl, $\mathrm{pH} 10,1 \%$ Triton $\mathrm{X}-100$ for $1 \mathrm{~h}$. After washing three times in AlkA buffer ( $40 \mathrm{mM}$ Hepes, 0.5 $\mathrm{mM}$ EDTA, $0.2 \mathrm{mg} / \mathrm{ml}$ bovine serum albumin, $\mathrm{pH}$ 8.0), the gels were incubated for times as indicated with $50 \mu \mathrm{l}$ of different concentrations of AlkA in this buffer at $37^{\circ} \mathrm{C}$. They were then treated for $40 \mathrm{~min}$ with $0.3 \mathrm{M} \mathrm{NaOH}, 1$ $\mathrm{mM}$ EDTA before electrophoresis at $0.8 \mathrm{~V} / \mathrm{cm}$ in this solution for $30 \mathrm{~min}$ at $4^{\circ} \mathrm{C}$. The gels were neutralised by washing three times in $0.4 \mathrm{M}$ Tris/ $\mathrm{HCl}, \mathrm{pH} 7.5$, and stained with DAPI. Visual scoring of comet images using fluorescence microscopy has been described (Collins et al., 1996). Each of 100 comets per gel is assigned to a category, from 0 to 4 , depending on the relative intensity of DNA fluorescence in the tail ( $0=$ undamaged, no DNA in tail: $4=>80 \%$ of DNA in tail). The total score for the gel, between 0 and 400, then represents the frequency of DNA breaks.

\section{RESULTS AND DISCUSSION}

The AlkA enzyme was prepared at different concentrations, and incubated for up to 
60 min with agarose-embedded nucleoids from freshly isolated lymphocytes. Results are shown in Fig. 1. The more concentrated

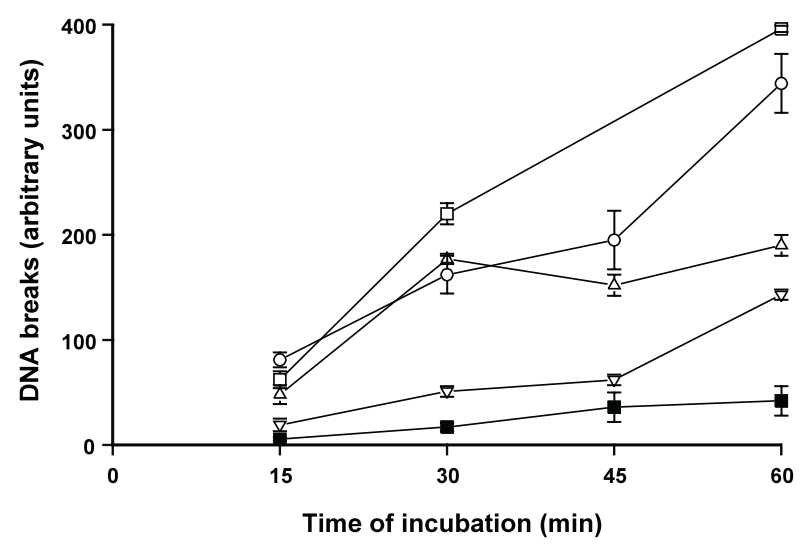

Figure 1. Activity of AlkA on DNA of untreated lymphocytes.

Effect of dilution by $100 \times(\square), 350 \times(O), 1000 \times(\Delta)$ and $3000 \times(\nabla)$. Control $(\mathbf{\square})$ no enzyme.

enzyme solutions induced DNA breaks that increased steadily with time. At a concentration of $1 / 1000$, breaks increased over the first 30 min of incubation but thereafter remained constant. At the lowest tested concentration, the breaks accumulated more slowly, as expected, and were still approaching the plateau level at $60 \mathrm{~min}$. Thus it seems that the more concentrated enzyme is able to react at sites in addition to those detected by the $1000 \times$ diluted enzyme. It is probable that the sites detected by the dilute enzyme are 3-methyladenine, while the more concentrated enzyme detects also damaged bases which are less favoured substrates for the enzyme. Or the additional sites might represent truly non-specific breakage by AlkA at undamaged bases, as reported by Berdal et al. (1998). While we cannot be sure of the precise identity of the sites detected by the dilute enzyme, they are almost certainly alkylated bases, which are of considerable biological interest as potentially mutagenic/carcinogenic lesions.

Lymphocytes from four individuals were compared for DNA breaks detected with AlkA at 1/1000 dilution. Incubation was for up to 60 min. A very similar pattern was observed in all four - as before, the break frequency increased over time, but reached a constant level at 30-45 min (Fig. 2). This plateau probably represents endogenous alkylation damage in the cellular DNA.

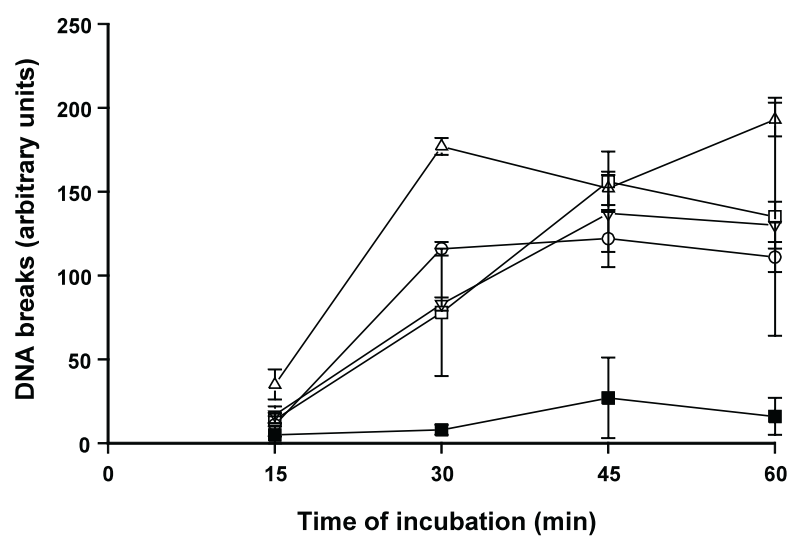

Figure 2. Activity of AlkA (1000 $\times$ diluted $)$ on DNA from untreated lymphocytes from four donors (open symbols).

The control incubation ( $\mathbf{\square}$ ) was with no enzyme. This figure includes data for one volunteer taken from Fig. 1 (1000× dilution).

To confirm that alkylation damage is detected by the enzyme under these conditions, isolated lymphocytes were treated with methyl methanesulphonate (MMS), which induces alkylation at various sites in cellular DNA. Figure 3 shows the MMS-dependent increase in breaks when these lymphocytes were treated after lysis with AlkA at 1/1000 dilution. At $0.1 \mathrm{mM}$ MMS, there are about 6 times as many AlkA-sensitive sites as strand breaks. Above $0.1 \mathrm{mM}$, the comet assay is insensitive to further increases in AlkA-sensitive sites, as a score of 400 indicates that all comets have maximal extension of DNA into the tail. However, the strand breaks (and alkali-labile sites) revealed in the absence of AlkA continue to increase with increasing MMS concentration.

Endogenous alkylation damage could occur by various mechanisms. For example, $S$-adenosyl methionine - the methyl donor acting as cofactor in many enzymatic reactions acts as a weak alkylating agent in its own 
right, causing damage to DNA (Lindahl, 1993).

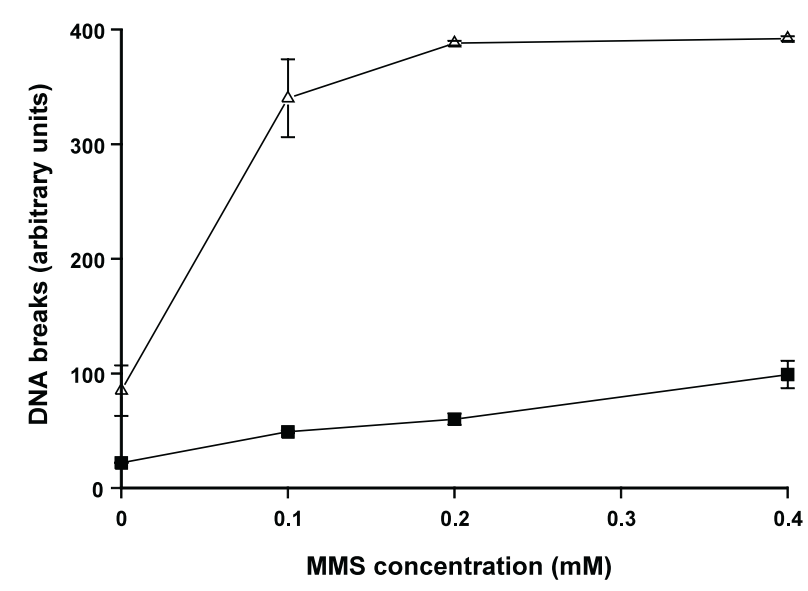

Figure 3. Detection of damage induced by alkylating agent MMS.

Lymphocytes were treated with MMS at the concentrations shown, for $15 \mathrm{~min}$, and then processed for the comet assay. Control ( $\square$ ), no enzyme; $(\Delta)$, plus AlkA at $1000 \times$ dilution, 45 min incubation.

Using a calibration based on X-irradiation of lymphocytes (Collins et al., 1996), we calculate that the level of alkylation damage detected with AlkA corresponds to about 0.8 sites per $10^{9}$ daltons of DNA, or 3000 alkylated bases per cell. This is very similar to the levels of oxidative base damage determined by comet assay with NTH and FPG (Collins et al., 1997a). We will now be able to study the range of intra- and inter-individual variation in background alkylation damage in humans, and investigate the possibility of modulation of this level of damage by environmental factors.

Preliminary work on this topic was carried out by Dr. Kateŕina Podrazilová.

\section{R E F E R E N C E S}

Berdal, K.G., Johansen, R.F. \& Seeberg, E. (1998) Release of normal bases from intact DNA by a native DNA repair enzyme. $E M B O J .17$, 363-367.

Collins, A.R., Dušinská, M., Gedik, C.M. \& Štetina, R. (1996) Oxidative damage to DNA: Do we have a reliable biomarker? Environ. Health Perspect. 104 (Suppl. 3) 465-469.

Collins, A.R., Duthie, S.J. \& Dobson, V.L. (1993) Direct enzymic detection of endogenous oxidative base damage in human lymphocyte DNA. Carcinogenesis 14, 1733-1735.

Collins, A.R., Duthie, S.J., Fillion, L., Gedik, C.M., Vaughan, N. \& Wood, S.G. (1997a) Oxidative DNA damage in human cells: The influence of antioxidants and DNA repair. Biochem. Soc. Trans. 25, 326-331.

Collins, A.R., Mitchell, D.L., Zunino, A., de Wit, J. \& Busch, D. (1997b) UV-sensitive rodent mutant cell lines of complementation groups 6 and 8 differ phenotypically from their human counterparts. Environ. Mol. Mutagen. 29, $152-160$.

Dušinská, M. \& Collins, A. (1996) Detection of oxidised purines and UV-induced photoproducts in DNA of single cells, by inclusion of lesion-specific enzymes in the comet assay. Altern. Lab. Anim. 24, 405-411.

Dušinská, M., Lietava, J., Olmedilla, B., Rašlová, K., Southon, S. \& Collins, A.R. (1999) Indicators of oxidative stress, antioxidants and human health; in Antioxidants in Human Health (Basu, T.K., Temple, N.J. \& Garg, M.L., eds.) pp. 411-422, CABI Publishing, Oxford, U.K.

Lindahl, T. (1993) Instability and decay of the primary structure of DNA. Nature 362, 709-715. 